\title{
Percepciones de Estudiantes de la Enseñanza de Inglés en cuanto al Aprendizaje de Idiomas y su Relación con el Género
}

\section{Leyla Tercanlioglu}

Departamento de Enseñanza de la Lengua Inglesa, Universidad de Atatürk, Erzurum

\section{Turquía}

Leyla@atauni.edu.tr 


\section{Resumen}

Introducción: Los estudiantes de idiomas extranjeros con frecuencia mantienen distintas creencias o ideas en cuanto al aprendizaje de idiomas (Horwitz, 1987), y la investigación existente sugiere que las creencias de los aprendices tienen la potencial de influir tanto en sus experiencias como en sus acciones como aprendices de idiomas.

Método: Este trabajo informa sobre un estudio que investigó las creencias sobre el aprendizaje de idiomas mantenidas por un grupo de estudiantes de la enseñanza de inglés, en cuanto se refería al género. Los participantes fueron 118 futuros profesores (45 varones y 73 mujeres), los cuales completaron el inventario de Horwitz, Beliefs About Language Learning Inventory [Creencias sobre el Aprendizaje de Idiomas], o BALLI.

Resultados: En el inventario BALLI, los participantes recibieron su puntuación más alta para el factor "motivaciones y expectativas de aprender un idioma". Los resultados demuestran una relación estrecha entre las variables. Los resultados del procedimiento ANOVA, para determinar diferencias relacionadas con el género, no indicaron ninguna diferencia significativa entre los varones y las mujeres.

Discusión: Los hallazgos de este estudio proporcionan una idea sobre sus creencias en cuanto a lo que más importa a la hora de aprender el inglés como idioma extranjero. Afirmaron que eran "las motivaciones y las expectativas de aprender". En segundo lugar, proporcionó una idea de las interrelaciones entre los factores de creencia: todos eran interrelacionados. Y en tercer lugar, proporcionó una idea de las relaciones entre los factores de creencias y el género: no se encontró ninguna diferencia significativa.

Palabras clave: creencias sobre el aprendizaje de idiomas, creencias de los aprendices, formación de profesores de inglés. 